University of Nebraska - Lincoln

DigitalCommons@University of Nebraska - Lincoln

April 1996

\title{
Spin polarization of the conduction bands and secondary electrons of $\mathrm{Gd}(0001)$
}

\section{Dongqi Li}

Materials Science Division, Argonne National Laboratory, Argonne, Illinois

J. Pearson

Materials Science Division, Argonne National Laboratory, Argonne, Illinois

\section{S.D. Bader}

Materials Science Division, Argonne National Laboratory, Argonne, Illinois

D.N. Mcllroy

University of Nebraska-Lincoln

C. Waldfried

University of Nebraska-Lincoln

See next page for additional authors

Follow this and additional works at: https://digitalcommons.unl.edu/physicsdowben

Part of the Physics Commons

Li, Dongqi; Pearson, J.; Bader, S.D.; Mcllroy, D.N.; Waldfried, C. ; and Dowben, Peter A., "Spin polarization of the conduction bands and secondary electrons of $\mathrm{Gd}(0001)$ " (1996). Peter Dowben Publications. 49.

https://digitalcommons.unl.edu/physicsdowben/49

This Article is brought to you for free and open access by the Research Papers in Physics and Astronomy at DigitalCommons@University of Nebraska - Lincoln. It has been accepted for inclusion in Peter Dowben Publications by an authorized administrator of DigitalCommons@University of Nebraska - Lincoln. 


\section{Authors}

Dongqi Li, J. Pearson, S.D. Bader, D.N. Mcllroy, C. Waldfried, and Peter A. Dowben 


\title{
Spin polarization of the conduction bands and secondary electrons of $\mathrm{Gd}(0001)$
}

\author{
Dongqi Li, J. Pearson, and S. D. Bader \\ Materials Science Division, Argonne National Laboratory, Argonne, Illinois 60439 \\ D. N. Mcllroy, C. Waldfried, and P. A. Dowben \\ Department of Physics, University of Nebraska, Lincoln, Nebraska 68588
}

\begin{abstract}
Angle- and spin-resolved photoemission was utilized to investigate the $5 d$ bulk bands and the surface state of $\mathrm{Gd}(0001)$ in the temperature range of $130-350 \mathrm{~K}$. The bulk bands at $1-2 \mathrm{eV}$ below the Fermi energy $E_{F}$ show Stoner-like behavior, while the temperature dependence of the surface state near $E_{F}$ indicates spin-mixing behavior due to fluctuating local $5 d$ moments. The secondary electron spectra of the Gd surfaces both before and after initial oxygen adsorption show a polarization dip at low kinetic energies due to the extra scattering channel for minority electrons via the unoccupied $4 f$ level. The temperature dependencies of the surface and bulk magnetization are separated using the spin polarization of the surface state and the bulk exchange splitting. (C) 1996 American Institute of Physics. [S0021-8979(96)21508-5]
\end{abstract}

Surface magnetism of the heavy rare earth metals has been the subject of great interest because of the unique phenomena exhibited. The Curie temperature $T_{C}$ of the $\mathrm{Gd}$ and Tb surfaces are reported to be significantly higher than that of the bulk. ${ }^{1-4}$ There is also evidence that the magnetic moments at the surface are canted out of the surface plane, ${ }^{3,5}$ while the bulk of the $\operatorname{Gd}(0001)$ films, $<400 \AA$ thick, have in-plane anisotropy. ${ }^{6}$ The enhanced surface magnetic order is believed to originate from the surface electronic structure, namely, a magnetic surface state near the Fermi energy $E_{F}$ located around the Brillouin zone center. ${ }^{7-9}$ The $5 d$ bulk bands appear in spin-integrated photoemission at binding energies of 1-2 eV and exhibit a temperature-dependent exchange splitting $\Delta_{b} \cdot 10,11$

The study of surface magnetic order depends on separating the surface signal from that of the bulk. This has been accomplished previously by comparing relatively surfacesensitive techniques, like spin-polarized low energy electron diffraction, ${ }^{1}$ spin-polarized secondary electron spectroscopy, ${ }^{3}$ to a bulk measurement, or taking advantage of the surface core level shift of the Gd $4 f$ levels. ${ }^{3-5}$ Even with these techniques, it is still difficult to unambiguously distinguish the surface and the bulk. In the present work, we use the spin polarization of a magnetic surface state as an indicator of the surface magnetic order, and $\Delta_{b}$ as the bulk indicator to separate the two. The temperature dependance of the exchange splitting itself is a very interesting issue, since theories for finite temperature magnetism of itinerant electron systems are less developed than ground state theories. In addition, we examine the spin polarization of the secondary electrons, both as an additional indicator of the magnetization and to understand the anomalous polarization dip at low energy. ${ }^{12}$

Spin-polarized photoemission experiments were performed on the U5 undulator beamline of NSLS at Brookhaven National Laboratory. The ultrahigh vacuum chamber is equipped with low energy electron diffraction and a hemispherical electron energy analyzer with a lowenergy spin detector. The details of the experimental setup are described elsewhere. ${ }^{13}$ The sample preparation followed previous procedures. ${ }^{10,14}$ The $\mathrm{W}(110)$ single-crystal substrate was cleaned by flashing and annealing in oxygen. The nominally 80 - $\AA$-thick epitaxial $\mathrm{Gd}(0001)$ films were deposited thermally onto the room-temperature substrate and subsequently annealed to $780 \mathrm{~K}$ to improve the structural ordering and magnetic properties. ${ }^{15}$ The chamber pressure remained $<5 \times 10^{-11}$ Torr during the process to ensure cleanliness. Films made by similar procedures are known to have single domains with in-plane magnetization and low coercivities. The spin polarization was measured in the remanent state after the sample was magnetized in-plane with a pulse field. The samples show no sign of hydrogen or carbon contamination, although the annealed films have a trace amount of oxygen that is equivalent to $<0.05 \mathrm{~L}\left(1 \mathrm{~L}=1 \times 10^{-6}\right.$ Torr s) oxygen exposure at room temperature. All photoemission spectra were taken at normal emission with the light incident at an angle of $65^{\circ}$. The photoemission spectra of the conduction bands were taken at $h \nu=32.7 \mathrm{eV}$ and the secondary electrons at $69.5 \mathrm{eV}$. The sample was biased to $-30 \mathrm{~V}$ to minimize the stray field effects.

Typical spin-polarized photoemission spectra at different temperatures are shown in Fig. 1. The peak near $E_{F}$ is the surface state of $\operatorname{Gd}(0001)$, which is responsible for the enhanced magnetic ordering of the Gd surface. ${ }^{7-9}$ This feature is strongly spin polarized at low temperature, and the polarization has the same sign as that of the occupied $4 f$ feature at $8.6 \mathrm{eV}$ binding energy (not shown in the figure). With increased temperature, the spin polarization of the surface state decreases and approaches zero, while the peak position and intensity show no change. The minority-spin counterpart of this state has been observed as an unoccupied state above $E_{F}$ with inverse photoemission. ${ }^{16,17}$

The bulk bands at 1-2 eV exhibit a different temperature dependence. At low temperature there are two well-defined peaks with opposite spin polarization. They are the majority and minority spin branches of the $5 d$ bulk band at $\Gamma$, separated by $\Delta_{b}$ (with the former at higher binding energy). Note that the sign of the spin polarization of the surface state is the same as that of the majority-spin bulk band. This confirms that the surface state is of majority spin character and that the surface couples to the bulk ferromagnetically ${ }^{5,18}$ instead of 


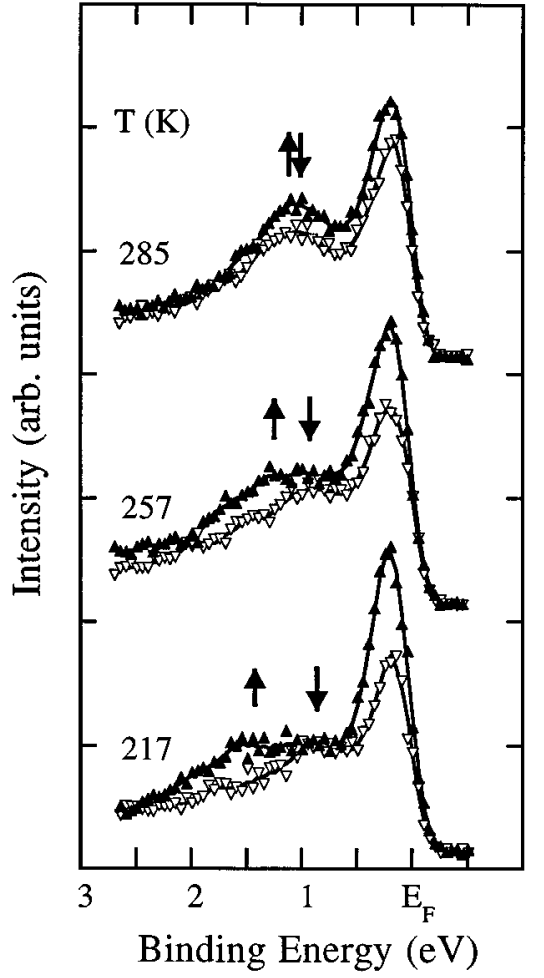

FIG. 1. Spin-polarized photoemission spectra at different temperatures at normal emission. The majority- and minority-spin components are shown with solid (up) and open (down) triangles, respectively. The majority- and minority-spin bulk bands are marked with up and down arrows. The solid lines are to guide the eye.

antiferromagnetically. ${ }^{1}$ With increased temperature the two peaks with distinct spin character shift toward each other and eventually overlap to form one peak with no spin polarization. In other words, $\Delta_{b}$ decreases and approaches zero upon warming to $T_{C}$.

The temperature dependence of the bulk bands is characteristic of Stoner-like ${ }^{19}$ behavior, where the exchange splitting $\Delta$ of the itinerant electrons directly correlates with the macroscopic magnetization and approaches zero as $T \rightarrow T_{C}$. The surface state, however, does not show an energy shift or intensity change near $T_{C}$, while the spin polarization varies significantly. This suggests that, instead of Stoner-like behavior, the local $\Delta$ of the surface state $\left(\Delta_{s}\right)$ does not go to zero at the surface Curie temperature $T_{\mathrm{Cs}}$. Such non-Stoner-like behavior is common among transition metals, although they were the original subject of the Stoner model. ${ }^{19}$ This is usually attributed to the existence of local moments or short range order above $T_{C}{ }^{20-22}$ We believe that the difference originates from the different degree of itinerancy of the electrons, as discussed elsewhere. ${ }^{14}$

The secondary electrons are also spin polarized, as shown in Fig. 2. The intensity of the secondary electron emission increases with oxygen exposure as oxygen atoms enhance the inelastic scattering in Gd. For the clean $\operatorname{Gd}(0001)$ surface, the spin polarization shows a dip when the kinetic energy of the electrons $E_{k}<1.5 \mathrm{eV}$. This polarization anomaly, first observed by Tang et al. ${ }^{12}$ is in contrast to the polarization increase at low $E_{k}$ for transition metal surfaces.

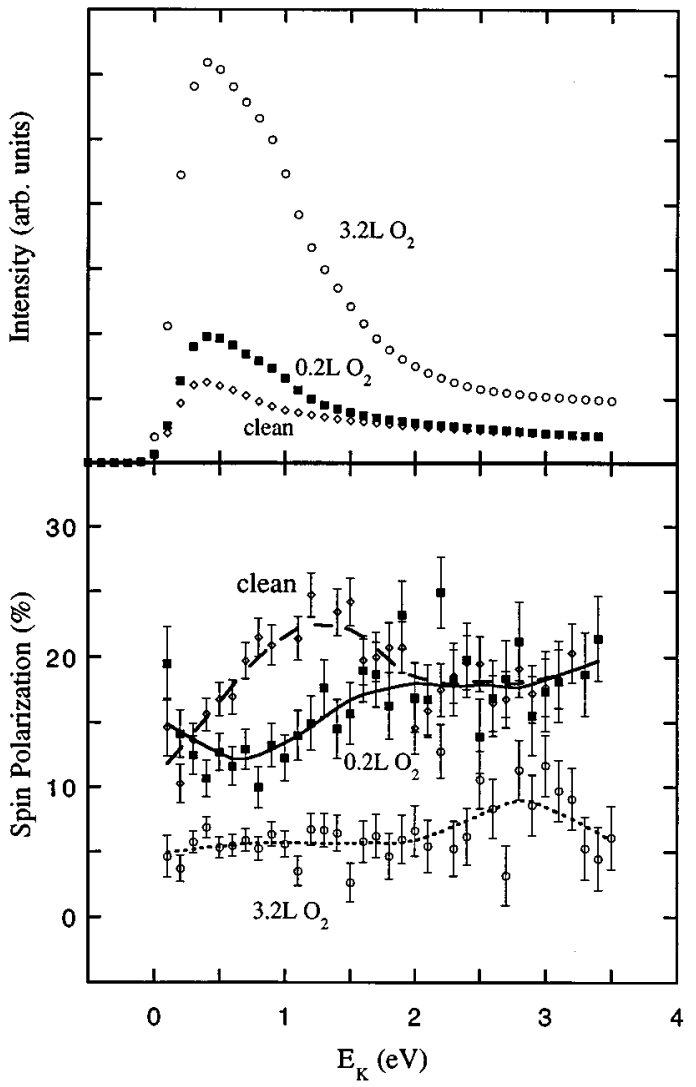

FIG. 2. Intensity and spin polarization of the secondary electron emission vs electron kinetic energy $E_{k}$. The measurements were taken at $130 \mathrm{~K}$ on clean $\mathrm{Gd}(0001)$ and after 0.2 and $3.2 \mathrm{~L}$ of oxygen exposure. The lines are to guide the eye.

Tang et al. suggested that an additional channel for the emission of minority spin electrons exists. The ordinary inelastic scattering of the hot electrons is determined by the unoccupied conduction bands, which provides the available states to scatter into. The unoccupied $4 f$ state above the vacuum level, i.e., $4 f,{ }^{8}$ could behave as an additional intermediate, where the minority hot electrons experience quasielastic scattering and emit to vacuum. This channel should selectively enhance the emission of minority spin electrons with $E_{k} \sim E_{4 f}-E_{v}$, where $E_{4 f}$ is the energy of the empty $4 f$ level above $E_{F}$ and $E_{v}$ is the vacuum level. For the clean $\mathrm{Gd}$ surface, the unoccupied $4 f$ level is at $4.1 \mathrm{eV}$ above $E_{F}$ and $\sim 0.8-0.9 \mathrm{eV}$ above $E_{v}$. Taking the width of the empty $4 f$ levels $(\sim 1.5 \mathrm{eV})^{23}$ into consideration, this possible mechanism explains the drop of spin polarization for $E_{k}<1.5 \mathrm{eV}$. Our data from the samples with initial oxygen adsorption supports such a hypothesis. Figure 2 shows that $0.2 \mathrm{~L}$ of oxygen exposure causes the onset of the spin-polarization dip to shift to higher energy by $\sim 0.6 \mathrm{eV}$. This is consistent with the shift of the empty $4 f$ level away from the $E_{F}$ with oxygen adsorption due to reduced screening. ${ }^{23}$ With $0.2 \mathrm{~L}$ of oxygen, the empty $4 f$ levels shift away from $E_{F}$ by $0.2-0.3$ $\mathrm{eV}$ (Ref. 23) while the work function drops by $0.2-0.3 \mathrm{eV}^{24}$ This results in the empty $4 f$ levels shifting up $\sim 0.5 \mathrm{eV}$ with respect to $E_{v}$, consistent within experimental error with the $0.6 \mathrm{eV}$ shift of the onset of the polarization dip. Other pos- 


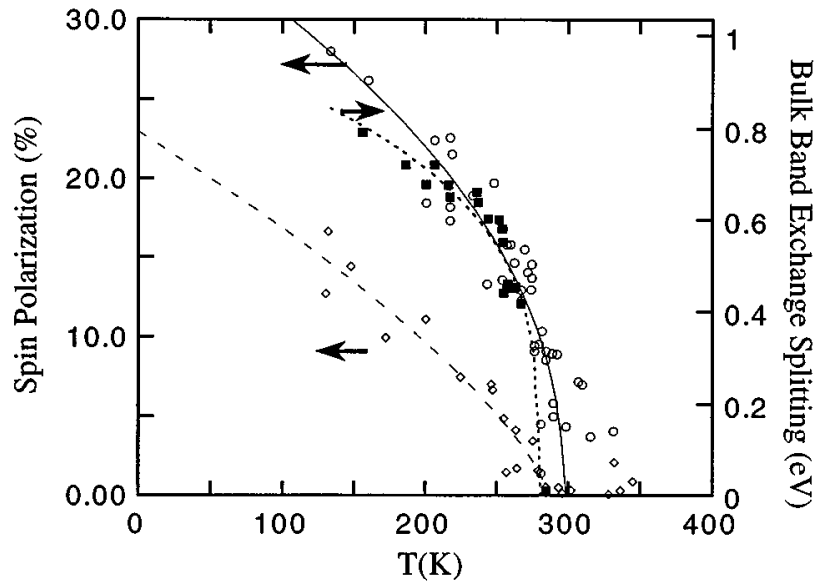

FIG. 3. Temperature dependence of the bulk band exchange splitting (squares), and the spin polarization of the surface state (circles) and secondary electrons (diamonds). The lines are to guide the eye.

sible scattering channels fail to provide the correct energy or the oxygen-induced shift of the dip. With additional oxygen dosing, the overall spin polarization drops further as the oxygen destroys the surface magnetic order of $\mathrm{Gd}$, and the dip disappears as both the $4 f$ and the valence band lose the net spin polarization. The effect of oxygen adsorption on the surface magnetization of $\mathrm{Gd}(0001)$ will be discussed further elsewhere. ${ }^{25}$

The spin polarization of the surface state and the secondary electrons and $\Delta_{b}$ are all correlated to the magnetization of the $\mathrm{Gd}(0001)$, though in somewhat different ways. The surface-state polarization reflects the purely magnetic order of the surface, and $\Delta_{b}$ reflects that of the bulk. The polarization of the secondary electrons should provide the mixed information from both the surface and the bulk. Figure 3 shows the temperature dependence of all three quantities. The spin polarization of the secondary electrons was measured at $E_{k}=3-4 \mathrm{eV}$ to avoid the anomaly at low energy, as discussed above. All quantities decrease with increasing temperature. The spin polarization of the secondary electrons and $\Delta_{b}$ approach zero before the surface-state polarization, as is consistent with $T_{\mathrm{Cs}}>T_{\mathrm{Cb}}$. This enhanced magnetic order can also be seen from Fig. 1, where $\Delta_{b}=0$ within experimental error, while the surface state and the background remain spin polarized. From Fig. 3, we find $T_{\mathrm{Cb}}=283 \pm 10 \mathrm{~K}$ and $T_{\mathrm{Cs}}=297 \pm 10 \mathrm{~K}$, with the accuracy limited by the signal-tonoise ratio.

In conclusion, we have studied the spin polarization of the conduction bands and secondary electrons of $\mathrm{Gd}(0001)$ at different temperatures. The $5 d$ bulk band shows Stoner-like behavior at the Brillouin zone center, while the surface state has a nonzero exchange splitting even above $T_{C}$. The anomaly in secondary electron polarization for both the clean and oxygen-adsorbed surfaces is discussed in terms of an extra scattering channel via the empty $4 f$ levels. The shift in the polarization dip to higher kinetic energy with initial oxygen adsorption is consistent with the chemical shift of the empty $4 f$ levels. In addition, our results are consistent with the enhanced magnetic order of the $\operatorname{Gd}(0001)$ surface.

We thank D.-J. Huang and Q. Dong for technical assistance and P. D. Johnson for valuable discussions. The work at Argonne is supported by DOE BES-MS Contract No. W-31-109-ENG-38 and ONR Contract No. N-00014-94-F0085; the work at Nebraska is supported by NSF Grant No. DMR-92-21655.

${ }^{1}$ D. Weller, S. F. Alvarado, W. Gudat, K. Schröder, and M. Campagna, Phys. Rev. Lett. 54, 1555 (1985).

${ }^{2}$ C. Rau, C. Jin, and M. Robert, Phys. Lett. A 138, 334 (1989).

${ }^{3}$ H. Tang, D. Weller, T. G. Walker, J. C. Scott, C. Chappert, H. Hopster, A. W. Pang, D. S. Dessau, and D. P. Pappas, Phys. Rev. Lett. 71, 444 (1993).

${ }^{4}$ E. Vescovo, C. Carbone, and O. Rader, Phys. Rev. B 48, 7731 (1993).

${ }^{5}$ D. Li, J. Zhang, K. Garrison, and P. A. Dowben, J. Phys. Condens. Matter 5, L73 (1993).

${ }^{6}$ A. W. Pang, A. Berger, and H. Hopster, Phys. Rev. B 50, 6457 (1994).

${ }^{7}$ D. Li, C. W. Hutchings, P. A. Dowben, C. Hwang, R.-T. Wu, M. Onellion, A. B. Andrews, and J. L. Erskine, J. Magn. Magn. Mater. 99, 85 (1991).

${ }^{8}$ D. Li, J. Zhang, P. A. Dowben, and M. Onellion, Phys. Rev. B. 48, 5612 (1993).

${ }^{9}$ R. Wu and A. J. Freeman, J. Magn. Magn. Mater. 99, 81 (1991).

${ }^{10}$ D. Li, J. Zhang, P. A. Dowben, and M. Onellion, Phys. Rev. B 45, 7272 (1992).

${ }^{11}$ B. Kim, A. B. Andrews, J. L. Erskine, K. J. Kim, and B. N. Harmon, Phys. Rev. Lett. 68, 1931 (1992).

${ }^{12}$ H. Tang, T. G. Walker, H. Hopster, D. P. Pappas, D. Weller, and J. C. Scott, Phys. Rev. B 47, 5047 (1993).

${ }^{13}$ P. D. Johnson, N. B. Brookes, S. L. Hulbert, R. Klaffky, A. Clarke, B. Sinkovic, N. V. Smith, R. Celotta, M. H. Kelly, D. T. Pierce, M. R. Scheinfein, B. J. Waclawski, and M. R. Howells, Rev. Sci. Instrum. 63, 1902 (1992).

${ }^{14}$ D. Li, J. Pearson, S. D. Bader, D. N. McIlroy, C. Waldfried, and P. A. Dowben, Phys. Rev. B 51, 13895 (1995).

${ }^{15}$ M. Farle, K. Baberschke, U. Stetter, A. Aspelmeier, and F. Gerhardter, Phys. Rev. B 47, 11571 (1993).

${ }^{16}$ D. Li, P. A. Dowben, J. E. Ortega, and F. J. Himpsel, Phys. Rev. B 49, 7734 (1994)

${ }^{17}$ A. V. Fedorov, K. Starke, and G. Kaindl, Phys. Rev. B 50, 2739 (1994).

${ }^{18}$ G. A. Mulhollan, K. Garrison, and J. L. Erskine, Phys. Rev. Lett. 69, 3240 (1992).

${ }^{19}$ E. C. Stoner, Proc. R. Soc. London Ser. A 154, 656 (1936).

${ }^{20}$ V. Korenman, J. L. Murray, and R. E. Prange, Phys. Rev. B 16, 4032 (1977).

${ }^{21}$ A. J. Pindor, J. Staunton, G. M. Stocks, and H. Winter, J. Phys. F 13, 979 (1983).

${ }^{22}$ H. Hasegawa, J. Phys. Soc. Jpn. 46, 1504 (1979).

${ }^{23}$ J. E. Ortega, F. J. Himpsel, D. Li, and P. A. Dowben, Solid State Commun. 91, 807 (1994).

${ }^{24}$ J. Zhang, D. Li, M. Onellion, and P. A. Dowben, Surf. Sci. 329, 177 (1995).

${ }^{25}$ D. N. McIlroy, C. Waldfried, D. Li, J. Pearson, S. D. Bader, D.-J. Huang, P. D. Johnson, R. F. Sabirianov, S. S. Jaswal, and P. A. Dowben (unpublished). 